\title{
Optimal autoregulation of the cellular metabolism
}

\author{
Georgij Detchev and A. Moskona \\ Biophysical Section, Institute of Biology, \\ Bulgarian Academy of Sciences, Sofia, Bulgaria
}

\begin{abstract}
KURZFASSUNG: Optimale Selbstregelung des Zellstoffwechsels. Der Stoffwechsel wird als harmonisches System von im Raum organisierten und in der Zeit koordinierten Reaktionen betrachtet. Ein solches System besitzt Eigenschaften, die für das System als ganzes charakteristisch sind, für eine einzelne Reaktion aber nicht zutreffen. Die biologischen Objekte sind als geregelte physikalisch-chemische Systeme zu betrachten, bei denen die "Geschwindigkeitskonstanten" der am Metabolismus teilnehmenden Reaktionen direkt von der Enzymaktivität abhängen. Wenn sich die Enzymaktivität verändert, ändert sich gleichzeitig die "Kontrolle“ des Systems. Es wird das normale Optimum, auf welches sich der Stoffwechsel einer Zelle einstellt, bestimmt; ferner werden die Gleichungen für Reaktionen angegeben, welche die "Kontrolle" des Systems so durchführen, daß das Optimum erreidht wird. Biologische Systeme sind selbstregelnde Systeme.
\end{abstract}

\section{INTRODUCTION}

The present work is an attempt to elaborate on a more general view of cell metabolism, i. e. on its energetics and on the regulation of energy flow within the cell. Cell metabolism is treated as a harmonious system of reactions rigidly organized in space and co-ordinated in time, possessing certain special qualities characteristic of it as a whole and not inherent to any single reaction. This investigation is based on the fact, that the cell is an open irreversible system, which incessantly exchanges mass and energy with the surrounding medium. In the treatment of the thermodynamics of irreversible processes, one has to bear in mind the kinetics of the system of biochemical reactions, considered as an self-regulatory system, the control of which is described by the equations of dynamic programming (BELLMaN 1957) and the maximum principle (Pontrjagin et al. 1961, Rosonoer 1961). Before entering into the problem considered it will be helpful to clarify the approach to be used.

Let us imagine that the evolution of a physico-dhemical system is given by the following equations:

$$
\begin{aligned}
& \frac{d x_{1}}{d t}=k_{11} x_{1}+k_{12} x_{2}+\ldots k_{1 n} x_{n}+f_{1}(t)=\frac{d_{i} x_{1}}{d t}+\frac{d_{e} x_{1}}{d t} \\
& \frac{d x_{2}}{d t}=k_{21} x_{1}+k_{22} x_{2}+\ldots k_{2 n} x_{n}+f_{2}(t)=\frac{d_{i} x_{2}}{d t}+\frac{d_{e} x_{2}}{d t} \\
& \frac{d x_{n}}{d t}=k_{n 1} x_{1}+k_{n 2} x_{2}+\ldots k_{m n} x_{n}+f_{n}(t)=\frac{d_{i} x_{n}}{d t}+\frac{d_{e} x_{n}}{d t}
\end{aligned}
$$


where $x_{1}(t), x_{2}(t), \ldots, x_{n}(t)$ are the concentrations of the substances taking part in the reactions.

$$
f_{1}(t)=\frac{d_{e} x_{1}}{d t} ; f_{2}(t)=\frac{d_{e} x_{2}}{d t} ; f_{n}(t)=\frac{d_{e} x_{n}}{d t}
$$

are the velocities with which the corresponding substances exchange with the surrounding medium, and $k_{11}, k_{12}, \ldots, k_{n n}$ are the velocity constants of reactions that are taking place.

We can write down the system as:

$$
\frac{d x_{j}}{d t}=f_{j}\left(x_{1}, x_{2}, \ldots, x_{n}, t\right) \quad(j=1,2, \ldots, n)
$$

Let us imagine further that the velocity constants of the system (1) cease to be constant and that we are able, within definite limits, to change them arbitrarily (for example, by adding or withdrawing a catalyst). In this case, they will turn into controlling parameters of the physico-chemical system (1) which, in this way, becomes a controlled object. The dependences (2) will be as follows:

$$
\frac{d x_{j}}{d t}=f_{j}\left(x_{1}, x_{2}, \ldots, x_{n}, k_{11}, k_{12}, \ldots, k_{n n}, t\right) \quad(j=1,2, \ldots, n)
$$

Since the controlling parameters $k_{11}, k_{12}, \ldots, k_{n n}$ are always limited in practice, they are performed for the inequalities

$$
\left(k_{i j}\right) \leqq M_{i j} \quad(i=1,2, \ldots, n ; j=1,2, \ldots, n)
$$

In the case we are able to vary the controlling parameters arbitrarily the question appears of how to choose these controls in order to achieve an aim that has been set forth in advance; it can be a maximal quantity of chemical production, minimal time of the reactionary processes, etc. The existence of the limitations (4) with the controlling parameters make it impossible to solve these problems by means of the classical variational principles. Insuperable difficulties in the case of their application has led to development of the powerful modern methods of the optimal regulation based on Bellman's principle of dynamic programming and Pontruagn's principle of maximum. They enable us (after having found the functional' ${ }^{1}$ of the system that corresponds to the set forth in advance and having taken into consideration (3) and (4)) to determine the controlling parameters $k_{11}, k_{12}, \ldots, k_{n n}$ as functions of the concentrations $x_{1}, x_{2}, \ldots, x_{n}$ specifying the state of the system:

$$
k_{i j}=k_{i j}\left(x_{1}, x_{2}, \ldots, x_{n}\right) \quad(i=1,2, \ldots, n ; j=1,2, \ldots, n)
$$

This means that if we can measure with appropriate apparatuses the concentrations of the reacting substances $x_{1}, x_{2}, \ldots, x_{n}$ in every moment $t$ and if we have at our disposal effective mechanisms to change the controlling parameters $k_{11}, k_{12}, \ldots, k_{n n}$ thus modelling the functions (5), then the system described in (3) will be optimized according to the stated functional. With different functionals corresponding to differ-

1 The conventional analysis deals with the minimization or maximization of function of $n$ variables $P\left(x_{1}, x_{2}, \ldots, x_{n}\right)$ while in the calculus of variations the problems contain functions of functions. To denote the scalar magnitude which depends on the functions, the term "functional" is used. The functional is a rule under which to a given number corresponds a function or a multitude of functions. 
ent aims, the functions (5) will be different, and this will lead to different structures of the modelling effective mechanisms worked out in such a way as the use of a control of the system would compel.

In biological objects with normal cell metabolism

$x_{1}(t), x_{2}(t), \ldots, x_{n}(t)$ are all metabolites taking part in it, and

$k_{11}, k_{12}, \ldots, k_{i j}, \ldots, k_{m n}$ are the velocity constants of the corresponding reactions. If we denote each separate reaction with a serial number $\varrho$, we will have $k_{1}, k_{2}, \ldots, k_{\varrho}, \ldots, k_{k}$ as

$k_{11}=k_{1} ; k_{12}=k_{2} ; k_{i j}=k_{0} ; k_{n n}=k_{k}$.

With the cell metabolism the velocity constants depend directly on enzyme activities. Since the latter enzymes change, these velocity constants cease to be constant, and they turn into controlling parameters, the network of the metabolism itself turning into a controlling object. Furthermore, the relations (4) do not only exist as a result of the fact that the velocities of the reactions cannot be infinitive, but they become complicated to a considerable degree, since in all pathways of the metabolism a definite number of enzymes play the role of specific regulators, which control the relative and absolute velocities. Thus among the regulatory parameters $k_{11}, k_{12}, \ldots$, $k_{n n}$ exist $l$ in number relations $\Psi$, which reflect the relations among the velocities of the metabolic processes:

$$
\Psi_{\beta}\left(k_{11}(t), k_{12}(t), \ldots, k_{n n}(t)\right) \leqq 0 \quad(\beta=1,2, \ldots, l)
$$

Let us imagine that we have found the normal natural functional which is optimized by the control of the metabolic network (we discern it from the functionals that are being optimized in some pathological states and with the specialized cells). Knowing the functional and the equations (4) and (6), we can find the relations

for the normal cell metabolism.

$$
k_{i j}=k_{i j}\left(x_{1}, x_{2}, \ldots, x_{n}\right)
$$

It was pointed out above that, knowing these functions (5), we can easily construct measuring instruments and effective mechanisms so that the controlled objects will be optimized. In its evolution nature has built up analogues of similar instruments and effectory mechanisms in biological objects. These are the enzymes whose activity is controlled by steric and allosteric effects or, in other words, the controlling parameters $k_{11}, k_{12}, \ldots, k_{n n}$ of the system change under the influence of the metabolites $x_{1}, x_{2}, \ldots, x_{n}$ taking part in the metabolism. Nature has created the enzymic apparatus of metabolism so that it would prove to model the functions (7). Since the metabolites $x_{1}, x_{2} \ldots x_{n}$ themselves regulate the parameters $k_{11}, k_{12}, \ldots, k_{n n}$ the biological systems are self-controlling or self-regulatory systems.

\section{AN ATTEMPT TO FIND THE NORMAL FUNCTIONAL OF THE NETWORK OF THE METABOLIC PROCESSES}

We shall start by defining the network of cellular metabolic processes in a thermodynamical aspect. (1) The elements of the network are: $x_{1}(t), x_{2}(t), \ldots \ldots x_{n}(t)$, intermediary and exchanging products, taking part in the metabolic reactions where $x_{j}(t)$ 
is the concentration of the $j$-metabolite in time $t$. (2) Two elements of the network a predecessor and a product of the $Q$-chemical reaction - will be denoted here a link in the network of metabolic processes. (3) Several links from the network of metabolic processes form a chain of reactions. (4) Every intermediary product, which is, in several metabolic processes, the initial substance, and, in others, the final one, takes part simultaneously in more than one chain; it will be denoted the node of the network of metabolic processes.

Let us assume that to every element of the network corresponds a chemical potential $\mu_{j}$, which has a definite value for a given substance and characterizes the free energy of one mole of the given substance.

Every node $g$ of the network of metabolic processes, limited by two elements of the network with chemical potentials $\mu_{1}$ and $\mu_{2}$, is characterized by a chemical affinity $A \varrho$. This latter is the motive force which causes the $\varrho$-elementary reaction to take place, and is equal to the difference between the chemical potentials $\mu_{1}$ and $\mu_{2}$ of the substances, taking part in the reaction with a stechiometric coefficient "one". The chemical affinity is characterized by the free energy released during the reaction. Every link of the network of the metabolic processes is characterized not only by the chemical affinity, but also by the velocity vo of the reaction taking place.

We shall now deal with two of the characteristic peculiarities of living systems, which will enable us to find the normal natural functional of cell metabolism. - The first peculiarity is the existence of conjugated reactions (KREBS \& KORNBERG 1957). By this term we denote those reactions which have a direction opposite to their own affinity and which are always realized with an increase of free energy. It is through them that the organisms synthesizes different chemical substances like ATP, creatine phosphate, proteins, lipids, etc.

The production of an inner entropy in an irreversible system, where $k$ reactions take place, depends on their intensity and is given by the expression (Prigogine 1955)

$$
T \frac{d i}{d t}=\sum_{\varrho=1}^{k} A \varrho v_{\varrho}=-\sum_{j=1}^{n} \frac{\delta G}{\delta x_{j}} \frac{d_{i} x_{j}}{d t}
$$

If in equation (8) we let the common destructive reactions of the biological oxidation marked with the indices 1 to $m$ and the conjugated reactions with the indices $m+1$ to $k$ grow, the entropy of the system will always decrease (in the conjugated reactions $A \varrho$ and $v \varrho$ are of opposite sign) and we shall get:

$$
T \frac{d_{i} S}{d t}=\sum_{\varrho=1}^{m} A \varrho v \varrho-\sum_{\varrho} \sum_{m}^{k} \quad A \varrho v \varrho=-\frac{d G_{1}}{d t}+\frac{d G_{2}}{d t}
$$

The quantity of free energy $G$ (GIBB's potential) is determined by the state parameters. At constant temperature and pressure $(T=$ const. and $p=$ const.), the independent state variables are momentary concentrations of all intermediary and exchanging metabolites. It follows from equation (9) that the changes of free energy $G\left[x_{1}(t), x_{2}(t) \ldots x_{n}(t)\right]$ for the whole system are due to the difference between $G_{1}$ released by the processes of oxidation and $G_{2}$ used by the organism through the conjugated reactions. 
The first thermodynamical principle allows us to find the energetic balances of the system, but it cannot demonstrate explicitly thermodynamic processes, since it does not convey any information of their realization and of the direction of their flow. It is evident that one and the same increase of the inner energy corresponds to a strictly fixed difference between heat consumed and work performed; however, the first principle of thermodynamics is not able to give the distribution of the energy between those two processes. Only the second principle of thermodynamics will give us the answer to these questions. In our irreversible system the every second production of entropy $T \frac{d_{i} S}{d t}$ in equation (9) is always higher than zero. Yet it is obvious that this is not sufficient to provide us with the distribution of energetic flows of the conjugated and the destructive reactions in equation (9) for the relation between the changes of $G_{1}$ and $G_{2}$. An attempt at solving the question can be done by taking into account the second characteristic peculiar of the biological systems.

As was already mentioned the results of a great number of biochemical investigations show that the reactions, which build up the network of the metabolic processes are controlled reactions (Chance et al. 1960; Holtzer 1963). The transmission and conversion of substances and energy rigidly co-ordinated in space and time, as well as their transport, accumulation and utilization are unthinkable without direct optimal regulation. This is based on a system of feedbacks, which realizes a constant exchange of information between the parts of the cell and between the cell and the surrounding medium. Through resulting functional organisation every disturbance of the steady state of the metabolic processes leads to a new steady state.

After having discussed these two peculiarities let us find the normal natural functional of cell metabolism. If we imagine that the system starts from an initial state $x^{0}, t_{0}$ and terminate its transient process after the time $T$ reaches a steady state, in which the thermodynamic potential has the value $G_{T}$. The latter will be a function of the variables $x_{1}(t), x_{2}(t), \ldots, x_{n}(t)$. Since their solutions (see Eq. 3) depend on $n+1$ initial conditions $x_{1}^{0}, x_{2}^{0}, \ldots, x_{n}^{0}$ and $t_{0}$, then $G_{T}$ will also depend on them, e. g. $G_{\eta^{4}}=G_{T}\left(x^{0}, t_{0}\right)$.

Let us single out, on the pathway of the system from $x^{0}, T^{0}$ to $x(T), T$ several points, for example, $x^{0}, t_{0} ; x^{\prime}, t^{\prime} ; x^{\prime \prime}, t^{\prime \prime}$, etc. We can consider each of them as an initial state of the system, which is always established in its final steady state with an thermodynamical potential $G_{T}$. We can write that down as

$$
G_{T}\left(x^{0}, t_{0}\right)=G_{T}\left(x^{\prime}, t^{\prime}\right)=G\left(x^{\prime \prime}, t^{\prime \prime}\right)=\text { const. }
$$

After having omitted the indices and instead of writing $G_{T}$ everywhere, we write down $G$, knowing that $G$ is function of the initial conditions; we can thus write:

$$
G(x, t)=\text { const., }
$$

from where

$$
\frac{d G}{d t}=\frac{\delta G}{\delta t}+\sum_{i} \frac{\delta G}{\delta x_{i}} \frac{\delta x_{i}}{d t}=0
$$

or 


$$
\frac{\delta G}{\delta t}=-\left(\sum_{j} \frac{\delta G}{\delta x_{j}} \frac{d_{i} x_{j}}{d t}+\sum_{j} \frac{\delta G}{\delta x_{j}} \frac{d_{e} x_{j}}{d t}\right)
$$

At time $t=T$ and $x=x(T), \quad \sum_{j} \frac{\delta G}{\delta x_{j}} \frac{d_{e} x_{j}}{d t}=\sum_{j} \frac{\delta G}{\delta x_{j}} f_{j}(T)=\Theta(T)$

characterizes the change of the free energy, depending directly on the time through the exchange of substances with the surrounding and $\sum_{j=1}^{n} \frac{\delta G}{\delta x_{j}} \frac{d_{i} x_{j}}{d t}$, the change of the free energy due to the running of the reactions into the system. We shall concentrate our attention on this sum, since it is the only one that depends on the controlling parameters.

Let us imagine for a moment that the cell were not able to organize itself functionally, e. g. the conjugated reactions do not exist and the whole quantity of free energy were dissipated as heat, which the organism is not able to use. Then we arrive at a merely open irreversible thermodynamic system, where $m$ reactions take place and about which from equation (9) we can put:

$$
T \frac{d_{i} S}{d t}=\sum_{\varrho=1}^{m} A \varrho v \varrho=-\frac{d G_{1}}{d t}=-\sum_{j=1}^{m^{\prime}} \frac{\delta G_{1}}{\delta x_{j}} \frac{d_{i} x_{j}}{d t}
$$

According to the well-known theorem that the flow of the irreversible processes in a thermodynamic system always diminishes the quantity of every second increase of entropy (Prigognne 1955), which in the steady state is minimum, it follows from equation (11) that:

$$
\frac{\delta G_{1}}{\delta t}=-\min \underset{j=1}{m^{\prime}} \frac{\delta G_{1}}{\delta x_{j}} \frac{d x_{j}}{d t}
$$

which is a trivial expression of the above-mentioned theorem.

Now let us imagine that the cell has begun functionally to organize itself, e.g. the conjugated reactions are starting and developing themselves. Then the useful flow, which is zero in equation (12), begins to increase. The system's organization will now become directed in such a way that under the concrete conditions this flow could be maximum. Thus the transitory process to a new steady state reveals the directiveness of the organization to the optimum - the new steady state. When we examine equation (9) from this point of view, we recognize that every second production of entropy decreases on account of two factors: (1) Decrease of the first sum in equation (9), characteristic of every irreversible system according to the theorem reflected in equation (13). (2) Increase of the second sum in equation (9), which "takes away" the entropy through the conjugated reactions involved in the processes of system organization or self-regulation.

If the system were not organized, but possessed conjugated reactions, then under the same concrete condition the last sum could assume different values. Thus, the steady state $T \frac{d_{i} S}{d t}$ in equation (9), in spite of the minimum of the first sum in equation (12), equation (13) would depend on these values and in the general case would not become minimum. 
There can be one single case in which every second production of entropy will be minimum - the case, when the second sum in equation (9) will be maximum, which is the goal of the whole functional organization of the cell. In this case and this case only, taking into consideration equations (8) and (9), we receive

$$
T \frac{d_{i} S}{d t}=\sum_{\varrho=1}^{m} A \varrho v \varrho-\sum_{\varrho} \sum_{+1} \quad A \varrho v \varrho=-\min \sum_{j=1}^{n} \frac{\delta G}{\delta x_{j}} \frac{d_{i} x_{j}}{d t}
$$

whereas for the common thermodynamic potential of the system $G$ from equations (11) and (14) we have:

$$
\frac{\delta G}{\delta t}=-\min \sum_{j=1}^{n} \frac{\delta G}{\delta x_{j}} \frac{d x_{j}}{d t}
$$

The resemblance between equations (13) and (15) is evident and therefore we shall draw our attention to their qualitative difference.

In every open irreversible thermodynamic system the simplest feedbacks can function, for instance, due to mass action law and to the velocities of the chemical reactions in the system etc. These relations are established with the participation of a considerable quantity of masses and energy. However, they have no informational character, which is why the systems in which they take place are usually uncontrolled. The system for which equations (12) and (13) apply is of this kind. In regulated systems flows of energy and substances are controlled by means of very small masses and energies; here the feedbacks and the controls bear an informational character. All biological systems are of this type.

In contrast to similar non-living systems, the minimizing of the general thermodynamic potential $G$ of the discussed system is due mainly to the maximum utilization of the energy $G_{2}$ by the organism as a result of its functional organization. Equation (15), in contrast to (13), reflects the organizing of the metabolic processes by regulation of the conjugation between the energy released by oxidative processes $G_{1}$ and that utilized by conjugated reactions $G_{2}$. Concrete biological experiments are obtained that prove the possibility of these flows (SKULATSCHEv 1961). Equation (15) simultaneously takes into consideration the degrees of the thermodynamical potential, which is characteristic of every irreversible system according to equations (12) and (13).

This analysis of equation (15) shows that it is entirely analoguous to BELLMAN's equation of dynamic programming, comprising all conclusions resulting from it. This analysis helps us to find the normal natural functional of the system. BeLLMaN's equation is an equation of partial derivatives, to avoid its solution we shall continue to use the system of ordinary differential equations, equivalent to it after PONTRJAGIN's maximum principle. In order to analyze them let us go further to the following construction. In every moment of time $t$ the network of metabolic processes will be determined by the concentrations of all the substances taking part in metabolism $x_{1}(t), x_{2}(t) \ldots x_{n}(t)$. If we consider these numbers as Cartesian coordinates in $n$ dimensional space, a determined point (vector) in it will correspond to every state of the network. Let us mark this $n$-dimensional space with $X$ and let us denote it conventionally the "space of metabolism". In the course of time the state of metabolic processes will alter and the index which depicts it will trace a determined trajectory in 
the space $X$. Let us suppose that in the moment $T_{0}$, the system is in its initial steady state in the point $x\left(T_{0}\right)$. After applying a defined influence (of physical or chemical character) in the moment $T$ the system will enter into its new stationary state $x(T)$.

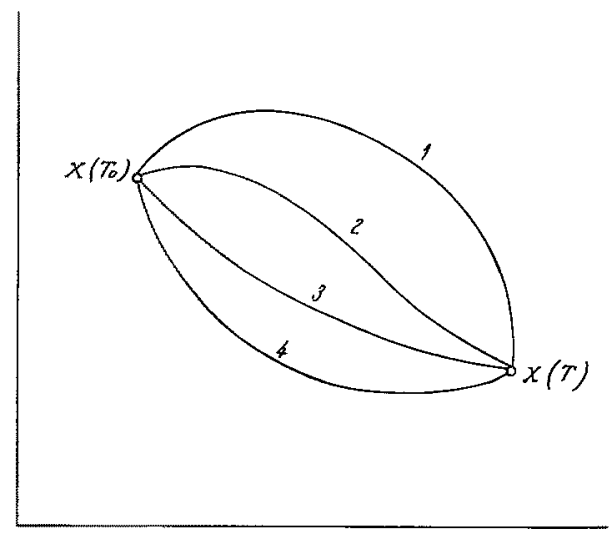

Fig. 1: Diagramm of four different trajectories

The path of the system from index $x\left(T_{0}\right)$ to index $x(T)$ may vary at great length, according to trajectories $1,2,3,4$, respectively (Fig. 1). The questions arise, which among all possible pathways between the two points will be realized by the system, on what will it depend and how will it vary through the different influences applied to the system.

\section{THE METABOLIC NETWORK AS AN OPTIMAL SYSTEM}

We try to answer these questions in the following way. In the terminology of the theory of the optimum processes, equation (15) shows that the functional, which minimizes, is the general thermodynamic potential $G\left(x_{1}, x_{2}, \ldots \ldots x_{n}\right)$ of the system. Under changed conditions, compared with the conditions of the initial steady state of the system $x\left(T_{0}\right)$, minimum holds at point $x(T)$ - the new stationary of the system. The consequent stage of minimizing of the functional to its value at point $x(T)$ determines simply the way of the system between the points $x\left(T_{0}\right)$ and $x(T)$. In order to follow this process, it is necessary to know the system's differential equations from $n$-th order, describing the examined object and its controling parameters - $k$ - whose change allows the functional of the system to minimize. Following the known kinetic peculiarities of the biochemical reactions, forming the network of the metabolic processes (PAssrnsky 1963), the system's equations of $n$-th order are formed through expression of the momentary changes of the concentrations of all metabolites taking part in the metabolism. The concentration $x_{j}(t)$ of the $j$-element in the network increases on account of all reactions for which $x_{j}(t)$ is the endproduct and decreases through all reactions for which $x_{f}(t)$ is an initial substrate, e. $g$. 


$$
\frac{d_{i} x_{j}}{d t}=\sum_{s} k_{s j} x_{s}-x_{j} \sum_{p} k_{j p} \quad(j=1,2,3 \ldots n)
$$

where $k_{s j}$ are the velocity constants of the reactions taking place from the $s$-th node to the $j$-th node, and $k_{j p}$ are the velocity constants of the reactions taking place from the $j$-th node to the $p$-th node.

The number of the equations (16) will be equal to the number of the elements in the system. If the reactions take place in an opposite direction, we will have for $\frac{d_{i} x_{j}}{d t}$

or generally for the node $x_{j}$ we get

$$
\frac{d_{i} x_{j}}{d t}=\sum_{p} k_{p j} x_{p}-x_{j} \sum_{s} k_{j s}
$$

$$
\frac{d x_{j}}{d t}=\sum_{s} k_{s j} x_{s}+\sum_{p}^{\sum} k_{p j} x_{p}-x_{j}\left(\sum k_{j p}+\sum_{p} k_{j s}\right)
$$

We shall continue to work with equation (16) because the corrections (17), on account of the reversible reactions in a biochemical system, are insignificant.

The controlling parameters in this case will again be the velocity constants $k_{s j}$ and $k_{j p}$ or $k_{g}$ if we denote with a general index the reactions $s \rightarrow j$ and $j \rightarrow p$. They will basically depend on the activities and the concentrations of the enzymes catalyzing the reactions between the elements $s$ and $j$ or the reaction of the link $\varrho$. The activity of enzymes in the cell changes even if very small changes among the conditions of the medium take place. Large intermolecular complexes are released from their structural steady state and begin to deaggregate - new active centres arise, which are accessible for interactions. This is proved by the increased adsorption power of the proteins (DetChev 1959). In this way the activity of enzymes as well as equation (6) can be changed. To clarify the physical interpretations, we shall examine the simpler case in a controlled closed system (on page 123 the general case in an open system has been considered), e. g.

$$
\frac{d x_{j}}{d t}=\frac{d_{i} x_{j}}{d t} \quad \text { hence } \quad \frac{d_{e} x_{i}}{d t}=f_{j}(t)=0
$$

Let us introduce the $k$-dimensional space $L$ whose co-ordinates are the $k$-control parameters of $k \varrho(t)$. For each combination of given values of the parameters, a respective point (vector) $k(t)$ in the space $L$ exists. It follows from physical considerations that the control $k(t)$ and its derivations are continous functions of time. The inequalities (6) determine in the space $L$ a closed manifold $K$ of points whose limits are fixed by the equations: $\Psi_{\beta}\left(k_{\varrho}\right)=0$. The control $k(t)$ at every moment $t$ must belong to the closed set $k$ of the space $L$. We shall call the control $k(t)$ belonging to $K$, i. e.

$$
k(t) \varepsilon K
$$

admissible. We shall discuss, together with the space $L$ of the control, the already introduced $n$-dimensional phase space $\left(x_{1}, x_{2} \ldots \ldots x_{n}\right)$ of the concentrations of the substances participating in the metabolism. If the control $k(t)$ and the initial conditions of the system $x^{0}\left(x^{0}{ }_{1}, x_{2}^{0} \ldots \ldots x_{n}^{0}\right)$ are given, then the trajectory $x(t)$ in the space $X$ is uniquely determined. In the further discussion we shall assume that no restrictions are imposed on the final point of the trajectory $x(t)$ in the phase space $X$, i. e. 
in the final state of the system, the vector $x(T)$ can embrace all the space $X$. The system will have a free right-hand end of its trajectory.

Let us introduce $n$-functions of time $p_{1}(t), p_{2}(t) \ldots p_{n}(t)$; they form the vector $p(t)=\left(p_{1}(t), p_{2}(t) \ldots \ldots p_{n}(t)\right)$ defined by the following differential equations:

$$
\begin{array}{r}
\dot{p}_{j}=-\sum_{n} p_{s} \frac{\delta \dot{x}_{s}\left(x_{1}, x_{2} \ldots x_{n} ; k_{1}, k_{2} \ldots \ldots k_{k}\right)}{\delta x_{j}} \\
(j=1,2, \ldots n)
\end{array}
$$

Let us introduce for each moment of time $t$ the function ${ }^{2}$ :

$$
H(x(t), p(t), k(t)) \equiv \sum_{j=1}^{n} p_{j} \frac{d x_{j}(x, k)}{d t}
$$

which we shall treat as a scalar product of the vector $p(t)$ with the vector of the system's velocity $\dot{x}(t)$. In analogy to analytical mechanics the function $H(x, p, k)$ is called Hamiltonian and the equations for $x_{j}(t), p_{j}(i)$ can be written as the canonical equations of HANiILTON:

$$
\dot{x}_{j}=\frac{\delta H}{\delta p_{i}} ; \quad \dot{p}_{j}=-\frac{\delta H}{\partial x_{j}} \quad(j=1,2 \ldots n)
$$

We shall say that the control $k(t)$ satisfies the conditions of the minimum if in every moment $t\left|T_{0} \leq t \leq T\right|$ the function $H(x, p, k)$ in which $x(t)$ and $p(t)$ are the position and the impulse of the image point in a moment $t$ respectively at control $k(t)$ is minimum, i. e. the function $M(k(t)) \equiv H(x(k), p(k), k)$, which depends on $k(t)$ only, reaches an absolute minimum in the multitude $K$ if the values of the variables equal the values of the control at the same time.

The maximum (minimum) principle of PONTRJAGIN will read thus: a control $k(t)$ will be minimum - (optimum) according to the functional $S$ composed by the lineal combination of the phase co-ordinates $x_{j}(t)\left|S=\sum_{j} c_{j} x_{j}(T)\right|$ when the control $k(t)$ satisfies the minimum conditions; in this definition we have put $p(t)=c$ (RosONOER 1961). Thus the optimum control $k(t)$ is chosen in every moment to minimize the function $H(x, p, k)$ or to minimize the scalar product of the vectors $p_{j}(t)$ and $\dot{x}_{j}(t)$.

The Hamiltonian in our system will be:

$$
\begin{gathered}
H=p_{1}(t) \dot{x}_{1}(t)+p_{z}(t) \dot{x}_{2}(t)+p_{3}(t) \dot{x}_{3}(t)+\ldots \\
\ldots \ldots+p_{n}(t) \dot{x}_{n}(t)
\end{gathered}
$$

substituting equation (16) in (22) we receive:

or we can transform:

$$
\begin{gathered}
H=p_{1}(t)\left(\sum_{s^{1}} k_{s^{1}} x_{s}-x_{1} \sum_{p^{1}} k_{1 p}\right)+p_{2}(t)\left(\sum_{s^{2}} k_{s^{2}} x_{s}-x_{2} \sum_{p^{2}} k_{2} p\right)+\ldots \\
\ldots+p_{n}(t)\left(\sum_{s^{n}} k_{s} n x_{s}-x_{n} \sum_{p^{n}}^{\Sigma} k_{n p}\right)
\end{gathered}
$$

$$
H=\left(\left(p_{1}-p_{2}\right) x_{1} k_{12}+\left(p_{1}-p_{3}\right) x_{1} k_{13}+\ldots-\left(p_{q}-p_{l}\right) x_{q} k_{q}\right)
$$

2 In general, $H$ may depend directly on time, but this does not change essentially the relations received (PONTRJAGIN et al. 1961, RosonOER 1961). 
From equation (15) and from the connection of Pontrjagrn's maximum principle with BeLLMAN's method of dynamic programming, two interesting conclusions can be drawn:

$$
\text { i) } \quad p_{j}(T)=\left.\frac{\delta G}{\delta x_{j}}\right|_{p, T}=\mu_{j}(T)
$$

i. e. that the chemical potentials of the metabolites, taking part in the metabolism are "impulses" of the controlled system and

$$
\text { ii) } \frac{\delta G}{\delta t}=H(x, p, k)=\sum_{j=1}^{n} p_{j} \frac{d x_{j}}{d t}
$$

i. e. that the Hamiltonian of the system is the momentary dissipation of the free energy.

We should, substituting the chemical potentials for the "impulses" in equation (23), come to a second conclusion; really,

$$
\begin{gathered}
H=\left(\left(\mu_{1}-\mu_{2}\right) x_{1} k_{12}+\left(\mu_{1}-\mu_{3}\right) x_{1} k_{13}+\ldots\right. \\
\left.\ldots-\left(\mu_{q}-\mu_{l}\right) x_{\mathrm{q}} k_{q} l\right)
\end{gathered}
$$

but, $\mu_{1}-\mu_{2}=A_{1}$ : the chemical affinity for the course of the first reaction (stoichiometric coefficients assumed as "one").

$\mu_{1}-\mu_{3}=A_{2}$ : the chemical affinity for the course of the second reaction

$\mu_{q}-\mu_{l}=A_{k}:$ the chemical affinity of the $k$-reaction

$x_{1} k_{12}=v_{1}:$ the velocity of the first reaction

$x_{1} k_{13}=v_{2}:$ the velocity of the second reaction

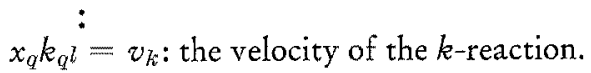

For $H$ we get:

$$
H=\left(A_{1} v_{1}+A_{2} v_{2}+\ldots .-A_{k} v_{k}\right)=\sum_{\varrho=1}^{k} A \varrho v
$$

which is another confirmation that the Hamiltonian of our system is the dissipation of free energy.

Let us write again the equation (16) in a more expanded form, keeping in mind that a part of the coefficients $k_{q^{l}}$ in the several equations are zero but we write them for the sake of symmetry.

$$
\begin{aligned}
& \frac{d x_{1}}{d t}=k_{11} x_{1}+k_{12} x_{2}+\ldots k_{1 n} x_{n} \\
& \frac{d x_{2}}{d t}=k_{21} x_{1}+k_{22} x_{2}+\ldots k_{2 n} x_{n} \\
& \vdots \\
& \frac{d x_{n}}{d t}=k_{n 1} x_{1}+k_{n 2} x_{2}+\ldots \ldots k_{n n} x_{n}
\end{aligned}
$$

The system (26) can be put in a vector-matrix form as:

$$
\frac{d x}{d t}=\dot{x}(t)=A x
$$


where the elements of the matrix $A$ are the velocity constants participating in (26):

$$
A=\left|\begin{array}{c}
k_{11} k_{12} \ldots \ldots k_{1 n} \\
k_{21} k_{22} \ldots . k_{2 n} \\
: \\
k_{n 1} k_{n 2} \ldots . k_{n n}
\end{array}\right|
$$

For the equations of the impulse, hawing in mind (19), (21) and differentiating (22) in $x_{j}$ we reveice:

$$
\begin{aligned}
& -\frac{d p_{1}}{d t}=k_{11} p_{1}+k_{21} p_{2}+\ldots+k_{n 1} p_{n} \\
& -\frac{d p_{2}}{d t}=k_{12} p_{1}+k_{22} p_{2}+\ldots+k_{n 2} p_{n} \\
& -\frac{d p_{n}}{d t}=k_{1 n} p_{1}+k_{2 n} p_{2}+\ldots+k_{n n} p_{n}
\end{aligned}
$$

or in vector-matrix form:

$$
\frac{d p}{d t}=p(t)=-\tilde{A} p
$$

where $\tilde{A}$ is the transponed matrix $A$ (28) which takes part in the equations (26) and (27).

\section{EQUATIONS WHICH DESCRIBE METABOLISM AS SELF-REGULATORY SYSTEM}

Suppose that the system has been at the point $x\left(T_{0}\right)$. Through an influence from outside the network of the metabolic processes is disturbed and begins immediately to organize itself according to equation (15) until it reaches the new point $x(T)$. The course of the system between these two points will simply be given by the following unified system of equations:

$$
\frac{d x}{d t}=A x \quad x^{0}=x^{0}\left(x_{1}^{0}, x_{2}^{0}, \ldots, x_{n}^{0}\right)
$$

equations expressing the kinetics of the biochemical reactions

$$
\frac{d p}{d t}=-\tilde{A} p \quad p^{0}=p^{0}\left(p^{0}{ }_{1}, p^{0}{ }_{2}, \ldots \ldots p_{n}^{0}\right)
$$

equations expressing the energetics of the biochemical reactions, received as a result of this investigation (at $t=T$ ) and finally

equations minimizing the Hamiltonian function $H(x(t), p(t), k(t))$, which unites the kinetics, the energetics as well as the control of our system.

From (31c) in the general case, by using the maximum principle we can receive for every $k=k(x, p)$, which, substituted in (31a) and (31b), gives a system of $2 n$ 
differential equations with $2 n$ unknown functions $x_{1}(t), x_{2}(t) \ldots x_{n}(t) ; p_{1}(t), p_{2}(t) \ldots$ $p_{n}(t)$. The solutions of this system at proper initial conditions will determine the trajectory of the system in the space of the metabolism $X$ and the development of the impulse $p(t)$. Knowing $x(t)$ and $p(t)$ we receive at every moment the control of the system $k=k(x(t), p(t))=k(t)$.

Let us first take the simplest case when no dependences occur between our parameters and equation (6) falls into separate inequalities for the parameters $k_{s j}$ and $k_{j p}$ of the type:

$$
\left|k_{s i}\right| \leq M_{s j}, \quad\left|k_{j p}\right| \leq M_{j \beta}
$$

The members of the Hamiltonian $H(x, p, k)$ of equation (23) fall into two groups with different signs. From the theorem of the maximum (minimum) it follows that in every moment $H$ must be minimum (p. 119). From the true character of the Hamiltonian, it is obvious that this can be realized at the expense of the decrease of the members of the first group (p. 115) and of the incessant increase of the members of the other group which corresponds to the increase of the conjugated reactions, $i$. e. the process of organization of the system (p. 116). Thus the theorem of the maximum (minimum) leads back to the thermodynamic premises at the basis of the present discussion.

Let at a given moment some of the constants $k_{\eta^{l}}$ become zero. At the same moment the matrix $\tilde{A}$ changes immediately because one of the elements forming it becomes zero. This consequently changes the system of linear differential equations (29), (30), the solution of which gives the development of the impulses in time. Therefore the new solutions of equations (29) and (30) will be defined already by the new matrix $\tilde{A}$ and the initial conditions, which will be the final values of the impulses received as solutions of the former system of equations at the moment of the "jump", i. e. at the moment of the change of the matrix $\vec{A}$ or at the moment of the disappearance of $k_{q}$. Thus we shall receive new developments of the impulses $p_{j}(t)$ in time. At a moment $t$ two of them, e. g. the $q$ and the r-ones $-p_{q}(t)$ and $p_{r}(t)$ - can become equal and then the reaction of this line will cease, the respective velocity constant will become zero, the matrix $\tilde{A}$ will change again, a "jump" will occur again, etc. Naturally, the matrix $A$ which takes part in the equations defining the momentous concentrations of the metabolites $x_{j}(t)$ (26) will change analogically determining their development in time. In the same way the conjugate reactions will develop, increasing instead of decreasing in the course of time. Thus the network of the metabolic processes will self-organize itself and its real development in time will be determined by the united system of equations (31).

In the living systems the maximum organization is reached due to feedbacks. In this case not all equations (6) dissipate into inequalities of the type (32), but a part of them, let us say $l$ relations, represents the feedbacks existing between the $r$ velocity constants in an implicit form. If all the velocity constants are as many as the reactions, i. e. $k$ then the independent control parameters will be $k-l$, and we shall receive for $k-r$ velocity constants (those both independent and taking no part in the relations $l$ ) the known result:

$$
|k|=\left|k_{i j}\right|=M_{i j} \operatorname{sign}\left(p_{i}-p_{j}\right)
$$


For the other $r-l$ independent parameters the equations of the type (33) will determine $k(t)$ as functions of $x$ and $p$, i. e. $k=k(x, p)$.

$$
\frac{\delta H}{\delta k}=0
$$

Thus in the cases when feedbacks in the system occur, the dependences (together with the respective initial conditions), which will determine the development, self-control and self-regulation of the network of the metabolic processes are the following:

$$
\begin{aligned}
& \text { a. } \frac{d x}{d t}=A x \quad x^{0}=x^{0}\left(x^{0}, x^{0}, \ldots x^{0}\right) \\
& \text { b. } \quad \frac{d p}{d t}=-\tilde{A} p \quad p^{0}=p^{0}\left(p^{0}{ }_{1}, p^{0}{ }_{2}, \ldots p^{0}{ }_{n}\right) \\
& \text { c. } \frac{\delta H}{\delta k}=0 \quad r-l \text { dependences } \\
& \text { d. } \Psi_{\beta}\left(k_{\varrho}\right)=0 \quad l \text { dependences } \\
& \text { e. }|k \varrho|=\left|k_{i j}\right| \leq M_{i j} \operatorname{sign}\left(p_{i}-p_{i}\right) \quad k-\mathrm{r} \text { dependences }
\end{aligned}
$$

After having considered the situation in a closed system, let us find how equations (31) would be in an open system. For the change of the reacting substances in the open system we can write:

$$
\begin{gathered}
\frac{d x}{d t}=\frac{d_{i} x}{d t}+\frac{d_{e} x_{j}}{d t}=\sum_{s} k_{j s} x_{s}-x_{j} \sum_{p} k_{j p}+f_{j}(t) \\
(j=1,2,3, \ldots n)
\end{gathered}
$$

where $f_{1}(t), f_{2}(t) \ldots f_{n}(t)$ characterize the exchange with the surrounding medium per unit of time, or in a vector-matrix form

$$
\frac{d x}{d t}=A x+f(t)
$$

Recalling equation (22) for the Hamiltonian of the system, we receive

$$
\begin{aligned}
& \mathrm{H}=p_{1}(t)\left(\sum_{s 1} k_{s 1} x_{s}-x_{1} \underset{p 1}{\sum} k_{1 p}+f_{1}(t)\right)+ \\
& +p_{2}(t)\left(\sum_{s 2}^{\sum} k_{s 2} x_{s}-x_{2} \underset{p 2}{\sum} k_{2 p}+f_{2}(t)\right)+\ldots \\
& \ldots+p_{n}(t)\left(\sum_{s n}^{\Sigma} k_{s n} x_{s}-x_{n} \underset{p n}{\Sigma} k_{n p}+f_{n}(t)\right)+ \\
& =\left(p_{1}-p_{2}\right) x_{1} k_{12}+\left(p_{1}-p_{3}\right) x_{1} k_{13}+\ldots-\left(p_{q}-p_{l}\right) x_{q} k_{q l}+ \\
& +p_{1}(t) f_{1}(t)+p_{2}(t) f_{2}(t)+\ldots+p_{n}(t) f_{n}(t)
\end{aligned}
$$

In this case $H$ depends not only on $x, p, k$ but also on the time $t$ (see footnote 1 on page 119). 
When minimizing the Hamiltonian with respect to $k_{\varrho}$, then it does not depend on the time $t$, and we receive again the well known result from the equations (34c).

The Hamiltonian equation (37) is zero in the steady state

$$
H(x, p, k, T)=0
$$

and together with the other equations we receive an equation, from which we can determine the time $T$, at which the system reaches its steady state.

Treating this case we have the following system of equations:

$$
\begin{aligned}
& \text { a. } \frac{d x}{d t}=A x+f(t) \quad x^{0}=x^{0}\left(x^{0}{ }_{1}, x^{0}{ }_{2}, \ldots x^{0}{ }_{n}\right) \\
& \text { b. } \frac{d p}{d t}=\tilde{A} p \quad p^{0}=p^{0}\left(p^{0}{ }_{1}, p^{0}{ }_{2}, \ldots p^{0}{ }_{n}\right) \\
& \text { c. } \frac{\delta H}{\delta k}=0 \\
& \text { d. } \quad H(x(T), p(T), k(T), T)=0
\end{aligned}
$$

From the investigations carried out in this paper we see, that taking into account the peculiarities of the biological objects, we have obtained the system of equations (39), which describes both the self-regulation of metabolism and the distribution of energetic flows in the cell.

\section{SOME GENERAL CONCLUSIONS}

By analyzing equations (29) and (31), the following conclusions can be drawn: In the space of metabolism the initial state is given by $x\left(T_{0}\right)$; the course of metabolism corresponds to the trajectory described by the system from point $x\left(T_{0}\right)$ to $x(t)$, the distance between the two depending mainly on the intensity of the acting factor. With very weak intensity the system compensates the changes due to the dynamical stability of the stationary state: $\left|x(t)-x\left(T_{0}\right)\right| \equiv 0$. With increasing intensity of the factor, completely reversible injuries occur and after cessation of the influence the network of metabolic processes return to the initial stationary state: $\left|x(t)-x\left(T_{0}\right)\right| \leqq R_{\text {irritation. With increasing intensity and duration of the influence }}$ the zone of stimulation and reversible injury is reached: $\left|x(t)-x\left(T_{0}\right)\right| \leqq R_{\text {stimulation, }}$, then the zone of irreversible injury: $\left|x(t)-x\left(T_{0}\right)\right| \leqq R_{\text {injury }}$ and finally death:

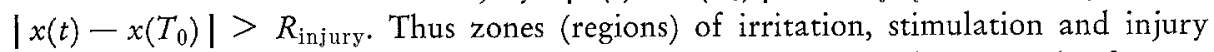
appear with the corresponding radii $(R)$, which depend upon the network of metabolic processes. The more intense the flow of metabolic processes, the more difficult to perform is its disorganization. The process of disorganization can easily be pursued in that the primary damage (element of the network) is still specific for an influencing factor, but if this brings about an ever expanding disorganization, it becomes less 
dependent on the specific properties of the factor and principally dependent on the genetically determined possibilities of the entire network. The process of disorganization (i. e. perpetual increase of the Hamiltonian) is counteracted by an opposite process (i. e. minimizing Hamiltonian), namely an organization resultant from regulation and feedback.

The concept developed briefly here has been experimentally checked with riceplants cultured on a thermogradient; growth vs. temperature-curve revealed an optimum; this indeed is enlarged in plants whose seedlings have been previously injured through treatment with tannin. Marked differences exist now in the amount of endoxidases between the untreated plants (at their temperature optimum) and the tannin-treated plants in the range of their optimum. This means that under the influence of the one and same compound i. e. tannin, two different enzyme apparatuses have been formed, obviously as result of autoregulation. The figures demonstrating these findings have been omitted from the paper. They will be published elsewhere.

\section{SUMMARY}

1. Metabolism is considered as a harmonious system of reactions rigidly organized in space and coordinated in time possessing properties characteristic of it as a whole but not specific for any single reaction.

2. An optimum of energetic flows of metabolic processes has been postulated. The network of metabolic processes is directed to it in such a way that the result provides a minimum dissipation of free energy in the system.

3. The treatment of this question is based upon the thermodynamics of irreversible processes in open systems and the mathematics of the dynamic programming after Bellman and of Pontrjagin's maximum principle.

4. The thermodynamic expression of the optimum to which the metabolism in a normally developed cell endeavours is derived.

5. A system of differential equations is formed, which reflects: (a) the formal kinetics of the biochemical reactions, (b) the energetics of the biochemical reactions, (c) the connection between the kinetics, the energetics and the control respectively of the system.

6. From the equations received the controls of the system, i. e. the activities of the enzymes, as well as of the concentrations of the participating metabolites, can be defined as functions of time.

7. In the interpretation of the above items, an attempt was made to extend the conventional thermodynamics of the irreversible processes so that it could include a regulatable physico-chemical system.

8. By introduction of several regions into the space of metabolism corresponding to different kinds of reactions, such as irritation, stimulation, injury and death, the different behaviour of the metabolic reactions with respect to the acting factor 
can be clearly demonstrated. Competion represents the motive power of the autoregulation, whereas the system of feedbacks is the apparatus of realization. Thus for given conditions the optimum organization can be reached.

\section{LITERATURE CITED}

Bellman, R. E., 1957. Dynamic programming. Princeton Univ. pr., Princeton, N. Y., 340 pp.

- Glicksberg, I. \& Gross, O. A., 1958. Some aspects of the mathematical theory of control processes.

CHANCE, B., 1961. Control characteristics of enzyme systems. In: Cellular regulatory mechanisms. Cold Spring Harb. Symp. quant. Biol. 26, 289-299.

- Carfinkel, D., Higgins, I. \& Hess, B., 1960. Metabolic control mechanisms. 5. A solution for the equations representing interaction between glycolysis and respiration in ascites cells. J. biol. Chem. 235, 2426-2439.

DantZig, G. B., 1964. New mathematical methods in the life sciences. Am. math. Mon. 71, $4-6$.

Detchey, G, 1956. Adsorption, Alteration, Denaturation und die dem Erregungsprozeß zugrunde liegenden Stoff wechselvorgänge. In: 2. Internationales Symposium über den Mechanismus der Erregung. Berlin, 31. 3.-2. 4. 1958. Hrsg. von J. Segal. Dt. Verl. d. Wissenschaften, Berlin (Fischer, Jena), 75-80.

- 1961. On the kinetics of the transition in new stationary states in plant organisms. [Russ.] Biofizika 6, 440-447.

- \& Moskona, A., 1964. Redox potential ob biological objects as result of irreversible processes in open systems. C. r. Acad. bulg. Sci. 17, 69-72.

Holtzer, H., 1963. Interzelluläre Regulation des Stoffwechsels. Naturwissenschaften 50, 260270.

KREBS, H. A. \& Korneng, H. L., 1957. A survey of the energy transformations in living matter. Ergebn. Physiol. 49, 212-298.

MittelstaedT, H., 1956. Regelungsvorgänge in der Biologie. Oldenbourg, München, 177 pp.

Passinsky, A. G., 1962. Some problems of biodhemical cybernetics. Vest. Akad. Nank SSSR $4,25-31$.

- 1963. Biophysical chemistry. [Russ.] Vaš̌aja škola, Moskva, 432 pp.

Pontruagin, L. S., Boltjansky, W. G., Gamkrelinse, P. W. \& Mischtenko, E. F., 1961. Mathematical theory of the optimal processes. [Russ.] Gosudarstv. Izd. fiziko-mat. Lit. Moskva, 392 pp.

PRIGOGINE, $\mathrm{Y}$., 1955. Introduction to thermodynamics of irreversible processes. C. C. Thomas, Springfield, Ill.

Rosonoer, L. I., 1961. Theory of optimal systems. [Russ.] Avomatika Telemekh. 20 (10), $1320(11), 1441(12), 1251$.

Skulatschev, W. P., 1961. About the regulation of the conjugation of oxydation and phosphorylation. Int. Congr. Biocbem. Moscow 5, 21-28. 


\section{Discussion following the paper by Detchev \& Moskona}

BRANSON: I have a small difficulty with your equation. If you write:

$\frac{d x_{i}}{d t}=A x_{i}$

$\frac{d p_{i}}{d t}=\tilde{A} p_{i}$

and $p_{i}=m_{i} \frac{d x_{i}}{d t}$, then

$\frac{d^{2} x_{i}}{d t^{2}}=A \frac{d x_{i}}{d t} \quad m_{i} \frac{d^{2} x_{i}}{d t^{2}}=A n \frac{d x_{i}}{d t}$ and $\frac{d p_{i}}{d t}=A p_{i}$

so $\mathrm{A}=\tilde{\mathrm{A}}$. That is carrying through the mechanical analogy.

Detchev: Die Funktionen $H$ und $P_{\mathrm{i}}$, die in der Theorie der optimalen Regulierung eingeführt werden, werden nur in Analogie mit der klassischen Mechanik "Hamiltonian" und "Impulse“ genannt. Aus diesem Grund ist der von Dr. Branson angedeutete Gedankengang unzulässig, um so mehr als sogar der Begriff "Masse" $\left(m_{1}\right)$ in der Theorie der optimalen Systeme nicht definiert ist.

HeinMETs: It can be questioned whether thermodynamic equations derived for solutions can be applied directly to cellular mediums containing density variations, interphases and aggregates.

Detchev: In den von uns angewandten Gleichungen steckt die Homogenität der Objekte weder in offenbarer, noch in versteckter Form; es ist die Kinetik, die bei der Betrachtung ähnlicher Probleme allgemein angewandt wird.

SUGITA: Is the maximum principle or the principle of minimum production of entropy of a thermodynamical nature or of a biological nature? There are oscillation phenomena. What might be the relation between the steady state consideration and the oscillation kinetics? Ordinary theory of irreversible processes is rather restricted, because only small deviation from equilibrium is considered or linear expansion is its foundation. Is there any consideration on this in using thermodynamical theory?

Detchev: Das Prinzip der minimalen Entropieproduktion ist in unserer Arbeit rein biologischer Natur. Bei dem hier angewandten Vorgehen wird das Verhältnis der Oszillationen zu dem stationären Zustand von dem Verhältnis der konkreten Werte der Koeffizienten bei Differentialgleichungen abhängen, so daß es im Augenblick schwer ist, im voraus darüber etwas zu sagen. Bei unseren Betrachtungen wird die starke Nichtlinearität der biologischen Objekte in Betracht gezogen. Gerade diese Tatsache hatte das Einführen einiger Elemente einer Thermodynamik geregelter irreversibler Prozesse zur Folge.

BalÁzs: Haben Sie die Einwirkung von niederen Temperaturen anstatt des Tannins auch untersucht?

DeTCHEv: Wir haben auch mit tieferen Temperaturen gearbeitet, aber ein Einfrieren nicht zugelassen - uns kam es nur auf eine Störung des Stoffwechsels an.

SCHARF: Die temperaturabhängige Wachstumsfunktion, die Herr DeTCHEv zeigte, dürfte in der vorliegenden Form nur für Pflanzen gelten. Bei Tieren fällt der rechte Schenkel wesentlich steiler ab, da zum Beispiel von Ratten höhere Temperaturen gewöhnlich schlecht vertragen werden.

Detchev: Unsere Theorie wurde auf pflanzlichem Experimentalmaterial ausgearbeitet. Die Grundlagen dürften aber auch für tierische Organismen gültig sein.

LOCKER: Ich kann mich nicht mit dem Gedanken befreunden, daß die von Ihnen kurz erwähnten Experimente eine Bestätigung Threr theoretischen Überlegungen sein sollten. Kurven, wie die von Ihnen gezeigten, lassen sich auf jeden Fall auch anders interpretieren und von einem gänzlich anderen Enzymbestand kann man bei den rein quantitativen Unterschieden, um die es sich hier bloß handelt, auch nicht sprechen. Die einzige exakte Möglichkeit, eine komplizierte 
Theorie, wie sie die Theorie des Dynamischen Programmierens nach Bellman und das PontrJAGINsche Maximal-Prinzip darstellen, in ihrer Anwendung auf biologische Phänomene zu rechtfertigen, also zu "beweisen", besteht darin, ein Modell zu bauen, das die durch die Theorie gegebenen Voraussagen erfüllt. Eine direkte Verifizierung, wie sie sich bei der so nützlichen Anwendung der Belimanschen Theorie auf Probleme der Raumschiffahrt ergibt, ist im biologischen Bereich nicht möglich. Wenn wir daran denken, daß die Theorie des Dynamischen Programmierens die Lösung von Problemen der Variationsrechnung ermöglicht, die ohne sie auch mit den schnellsten und größten Computern nicht gelöst werden können, drängt sich der Gedanke auf, sie selbst zur Grundlage von Computer-Experimenten zu nehmen, also genau den Weg zu beschreiten, den Dr. Hernmets, wenn auch von ganz anderen theoretischen Voraussetzungen aus, geht. - Im übrigen erscheint mir die Beschreibung der zellulären Autoregulation auf der Grundlage neuer mathematischer Verfahren, die bisher außerhalb der Technik meines Wissens noch sehr selten Anwendung fanden, sehr begrüßenswert.

MARMASSE: It must be pointed out that one has to be cautious in the interpretation in the contest of Dr. DeTchev's theory with respect to redox measurements because: several steps can sometimes be observed.

Detchev: Die Messung des Redox-Potentials bietet gegenüber photometrischen Messungen den Vorteil, integrale Werte über den Zustand der Zelle zu liefern. 